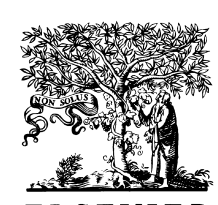

ELSEVIER

International Journal of Pressure Vessels and Piping 77 (2000) 3-16

Pressure Vessels

and Piping

\title{
Probabilistic analysis of off-center cracks in cylindrical structures
}

\author{
S. Rahman*, G. Chen ${ }^{\mathrm{a}}$, R. Firmature ${ }^{\mathrm{b}}$ \\ ${ }^{a}$ Department of Mechanical Engineering, The University of Iowa, Iowa City, IA 52242, USA \\ ${ }^{\mathrm{b}}$ IBM Printing Systems Company, Boulder, CO 80301, USA
}

Received 24 June 1999; accepted 28 October 1999

\begin{abstract}
This paper presents a probabilistic methodology for fracture-mechanics analysis of off-center cracks in pipes subject to pure bending moment. It is based on: (1) a new analytical approximation of the $J$-integral; (2) statistical models of uncertainties in loads, material properties, and crack geometry; and (3) standard computational methods of structural reliability theory. The proposed analytical equations were applied to a probabilistic fracture-mechanics analysis of off-center cracks in pipes. The second-order reliability method was used to determine the probabilistic characteristics of the $J$-integral and failure probability based on the initiation of crack growth. Numerical examples are presented to illustrate the proposed methodology. The results show that the failure probability strongly depends on the offcenter crack angle and is generally lower than that of a pipe with a symmetrically centered crack. Hence, simplifying an off-center crack by a symmetrically centered crack can produce significant conservatism in predicting failure probabilities. In addition, uncertainty in the offcenter crack angle, if it exists, can increase the failure probability of pipes. (C) 2000 Elsevier Science Ltd. All rights reserved.
\end{abstract}

Keywords: J-integral; Probabilistic fracture mechanics; Off-center crack; Pipe; Through-wall crack; Leak-before-break; J-estimation method

\section{Introduction}

Probabilistic elastic-plastic analysis of circumferential cracks in pipes is an important task for leak-before-break (LBB) $[1,2]$ and pipe flaw evaluations [3]. To predict the probability of fracture under external loads (e.g. bending or combined bending and tension loads), the crack-driving force, such as the $J$-integral, is typically evaluated by assuming that these cracks are symmetrically placed with respect to the bending plane of the pipe. This is usually justified by reasoning that the tensile stress due to bending is largest at the center of this symmetric crack. However, in reality, fabrication imperfections occur randomly around the pipe circumference. Additionally, during the normal operating condition of a power plant, the stress component due to pressure is far more significant than that due to bending. As such, the postulated flaw in LBB analysis may be off-centered [see Fig. 1] and can thus be located anywhere around the pipe circumference. The likelihood of a crack being off-centered can be further emphasized in light of the argument that a symmetric bending plane under normal operating stress may become very different under normal plus safe-shutdown earthquake stress, due to the

\footnotetext{
* Corresponding author. Tel.: +1-319-335-5679; fax: +1-319-335-5669

E-mail address: rahman@icaen.uiowa.edu (S. Rahman).
}

uncertainty in the seismic ground motion [4-6]. Hence, analysis of off-center cracks is an exciting research area for pipe fracture and LBB applications.

Previously, Rahman and Firmature [7] performed a deterministic study in which they developed new elastic and elastic-plastic finite element solutions of $J$-integral for offcentered through-wall cracks in pipes under pure bending. The analyses were performed for a wide variety of crack sizes, off-center crack angles, and material hardening exponents. Based on the results of this study, the crack-driving force and hence, the structural integrity of off-centered cracked pipes can be estimated. However, this was a strictly deterministic study. Due to inherent statistical variabilities in loads, material properties, and crack geometry, ultimately a probabilistic methodology is needed to evaluate the stochastic characteristics of fracture response and reliability of cracked pipes. Hence, the development of a probabilistic methodology for off-center cracks in pipes is both timely and exciting.

This paper presents a probabilistic methodology for fracture-mechanics analysis of off-center cracks in pipes subject to pure bending moment. It is based on: (1) analytical approximations of $J$-integrals calculated by elasticplastic finite element method (FEM); (2) statistical models of uncertainties in loads, material properties, and crack geometry; and (3) standard computational methods of structural reliability theory. The results from past finite 


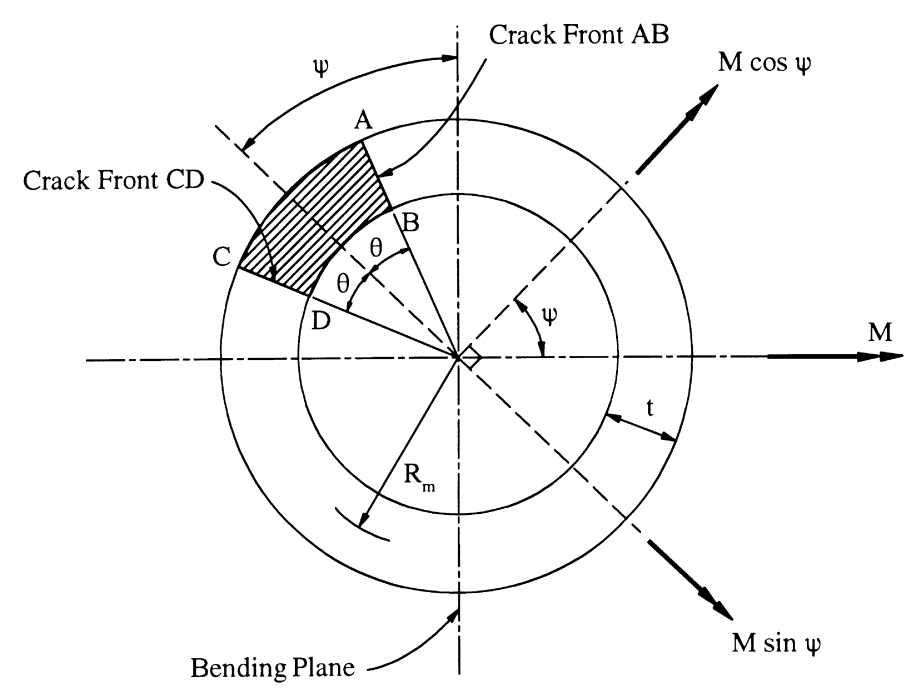

(a)

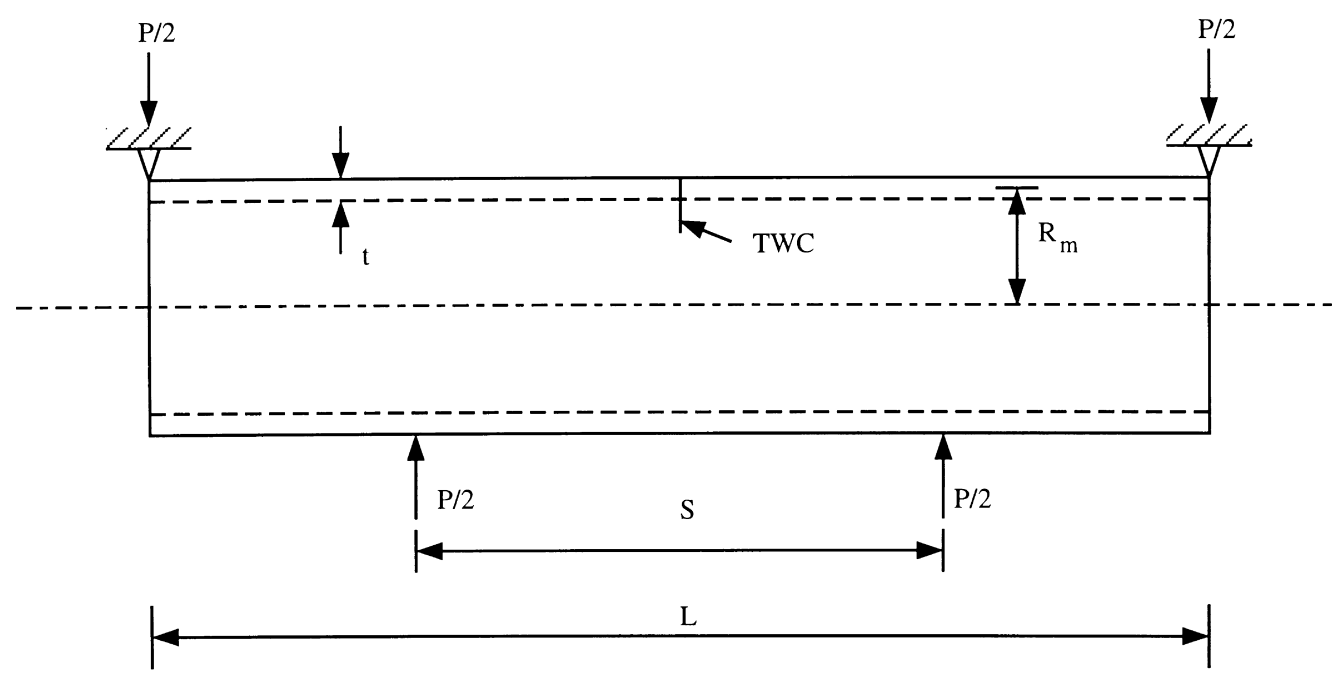

(b)

Fig. 1. A simple through-wall crack in a pipe under pure bending (a) off-center crack; (b) four-point loads simulating pure bending.

element analysis (FEA), performed for a wide variety of crack sizes, off-center angles, and material hardening parameter, were used to develop elastic and plastic correction factors for predicting $J$-integral. Following the response surface approximations of these correction factors, new closed-form analytical equations were developed for the $J$-integral. These analytical equations were then subsequently applied for probabilistic fracture-mechanics analysis. Both fast probability integrators, such as first- and secondorder reliability methods and simulation methods, such as Monte Carlo simulation and Importance Sampling, were used to determine the probabilistic characteristics of the $J$-integral. The same methods were used later to predict the failure probability based on the initiation of crack growth. A numerical example is presented to illustrate the proposed methodology.

\section{A TWC pipe with an off-center crack}

Consider a TWC pipe with mean radius, $R_{\mathrm{m}}$, wall thickness, $t$, and a through-wall-crack angle, $2 \theta$. The crack is offcentered by an angle, $\psi$. The pipe is subjected to a pure bending moment, $M$, without any internal pressure [Fig. 1(b)]. The geometric parameters of this off-center crack in the cracked section are defined in Fig. 1(a).

In order to perform an elastic-plastic analysis, the material model needs to be defined. In this study, it was 
Table 1

Matrix of finite element analyses for off-center cracks (five runs per model)

\begin{tabular}{|c|c|c|c|}
\hline FEM model no. & $\theta / \pi$ & $\psi\left({ }^{\circ}\right)^{\mathrm{a}}$ & $n^{\mathrm{b}}$ \\
\hline 1 & $1 / 16$ & 0 & $1,3,5,7$, and 10 \\
\hline 2 & $1 / 16$ & 15 & $1,3,5,7$, and 10 \\
\hline 3 & $1 / 16$ & 30 & $1,3,5,7$, and 10 \\
\hline 4 & $1 / 16$ & 45 & $1,3,5,7$, and 10 \\
\hline 5 & $1 / 16$ & 60 & $1,3,5,7$, and 10 \\
\hline 6 & $1 / 16$ & 75 & $1,3,5,7$, and 10 \\
\hline 7 & $1 / 16$ & 90 & $1,3,5,7$, and 10 \\
\hline 8 & $1 / 8$ & 0 & $1,3,5,7$, and 10 \\
\hline 9 & $1 / 8$ & 15 & $1,3,5,7$, and 10 \\
\hline 10 & $1 / 8$ & 30 & $1,3,5,7$, and 10 \\
\hline 11 & $1 / 8$ & 45 & $1,3,5,7$, and 10 \\
\hline 12 & $1 / 8$ & 60 & $1,3,5,7$, and 10 \\
\hline 13 & $1 / 8$ & 75 & $1,3,5,7$, and 10 \\
\hline 14 & $1 / 8$ & 90 & $1,3,5,7$, and 10 \\
\hline 15 & $1 / 4$ & 0 & $1,3,5,7$, and 10 \\
\hline 16 & $1 / 4$ & 15 & $1,3,5,7$, and 10 \\
\hline 17 & $1 / 4$ & 30 & $1,3,5,7$, and 10 \\
\hline 18 & $1 / 4$ & 45 & $1,3,5,7$, and 10 \\
\hline 19 & $1 / 4$ & 60 & $1,3,5,7$, and 10 \\
\hline 20 & $1 / 4$ & 75 & $1,3,5,7$, and 10 \\
\hline 21 & $1 / 4$ & 90 & $1,3,5,7$, and 10 \\
\hline
\end{tabular}

a $\psi=0$ implies symmetrically centered crack.

${ }^{\mathrm{b}} n=1$ implies linear-elastic analysis $(\alpha=0)$.

assumed that the constitutive law characterizing the material's stress-strain $(\sigma-\epsilon)$ response can be represented by the well-known Ramberg-Osgood model, which is

$\frac{\epsilon}{\epsilon_{0}}=\frac{\sigma}{\sigma_{0}}+\alpha\left(\frac{\sigma}{\sigma_{0}}\right)^{n}$,

where $\sigma_{0}$ is the reference stress which can be arbitrary, but is usually assumed to be the yield stress, $E$ is the modulus of elasticity, $\epsilon_{0}=\sigma_{0} / E$ is the associated reference strain, and $\alpha$ and $n$ are the model parameters usually chosen from a best fit of actual laboratory data. Although this representation of the stress-strain curve is not necessary for the finite element analysis, it is needed for most $J$-estimation methods, which are formulated based on power-law idealization.

\section{Past finite element analyses}

In the past, Rahman and Firmature performed 105 finite element analyses for calculating the $J$-integral for off-center cracks in a pipe with $R_{\mathrm{m}}=50.8 \mathrm{~mm}$ ( $2 \mathrm{in}$.) and $t=$ $5.08 \mathrm{~mm}$ (0.2 in.) [7]. A matrix of such analyses is defined in Table 1 for various combinations of the crack size, crack orientation, and strain hardening exponent: $\theta / \pi, \psi$, and $n$. It involves 21 different finite element meshes with $\theta / \pi=$ $1 / 16,1 / 8$, and $1 / 4$ and $\psi=0,15,30,45,60,75$, and $90^{\circ}$. For each mesh, five analyses were performed due to five different hardening exponents: $n=1,3,5,7$, and 10. For other material properties, the following values were used: $E=207 \mathrm{GPa}, \nu=0.3, \sigma_{0}=344.8 \mathrm{MPa}$, and $\alpha=0$ when $n=1$ and $\alpha=1$ when $n>1$. These values, in addition to the ones given in Table 1, provide complete characterization of the pipe material properties according to Eq. (1). All analyses were performed using the commercial finite element code ABAQUS [8].

Fig. 2(a)-(d) show the results of $J$ vs. $M$ plots from an elastic-plastic analysis with $n=5$ obtained for small $(\theta / \pi=1 / 16)$ and large $(\theta / \pi=1 / 4)$ cracks, respectively, at crack fronts $\mathrm{AB}$ and $\mathrm{CD}$ [see Fig. 1(a)]. The analyses involved various off-center crack angles with $\psi=0,15$, $30,45,60,75$, and $90^{\circ}$. The results indicate that the $J$-integral values at the two crack fronts of an off-center crack are unequal due to the loss of symmetry with respect to the bending plane of the pipe. In addition, the $J$-integral is larger, and hence, critical at the crack front which is farther away from the bending axis of the pipe. This is because, at that crack front, the tensile stress is larger and the component of the applied bending moment about the crack centerline has a further crack-opening effect. Also at this crack front, the $J$ values can be lower or slightly higher than those of a symmetrically centered crack, depending on the crack size and off-centered angle. For the crack front that is closer to the bending axis, the $J$ values are always lower than those of a symmetrically centered crack. This implies that the load-carrying capacity of a pipe is usually larger for an off-center crack than that for a symmetrically centered crack. See Rahman and Firmature [7] for further details on these finite element calculations.

The finite element results described above should be useful in developing analytical expressions of $J$-integral for fracture analysis of pipes containing off-center cracks. These analytical expressions will allow both deterministic and probabilistic pipe fracture evaluations without the need to perform a full-scale nonlinear finite element analysis. They are described in the next section.

\section{A new J-estimation method}

Under elastic-plastic conditions and applying the deformation theory of plasticity when the stress-strain curve is modeled by Eq. (1), the total crack driving force, $J$, for an off-centered crack of angle $\psi$ can be obtained by adding the elastic component, $J_{e, \psi}$, and the plastic component, $J_{p, \psi}$, i.e.

$J=J_{\mathrm{e}, \psi}+J_{\mathrm{p}, \psi}$

Closed-form equations already exist for the elastic and plastic components of a symmetrically centered crack [9-11]. It is proposed to use these equations along with simple off-center-angle correction factors for the calculation of $J_{\mathrm{e}, \psi}$ and $J_{\mathrm{p}, \psi}$. Hence, Eq. (2) can be re-written as

$J=J_{\mathrm{e}, 0} K_{\mathrm{e}, \psi}+J_{\mathrm{p}, 0} K_{\mathrm{p}, \psi}$

where

$J_{\mathrm{e}, 0}=\frac{\theta}{\pi} F\left(\frac{\theta}{\pi}, \frac{R_{\mathrm{m}}}{t}\right)^{2} \frac{M^{2}}{E R_{\mathrm{m}}^{3} t^{2}}$ 


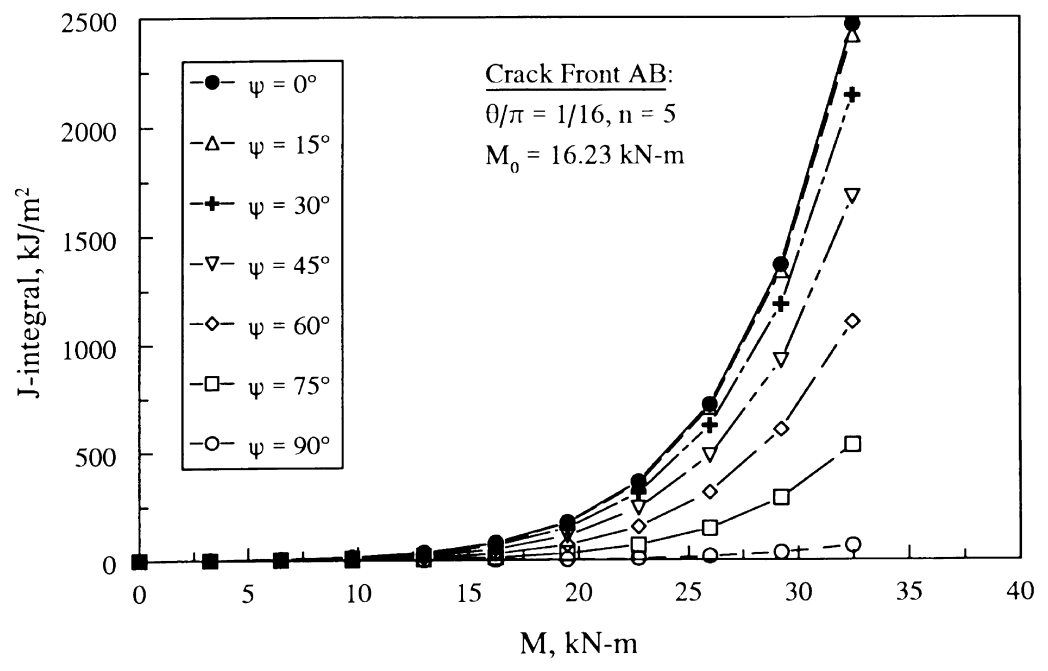

(a)

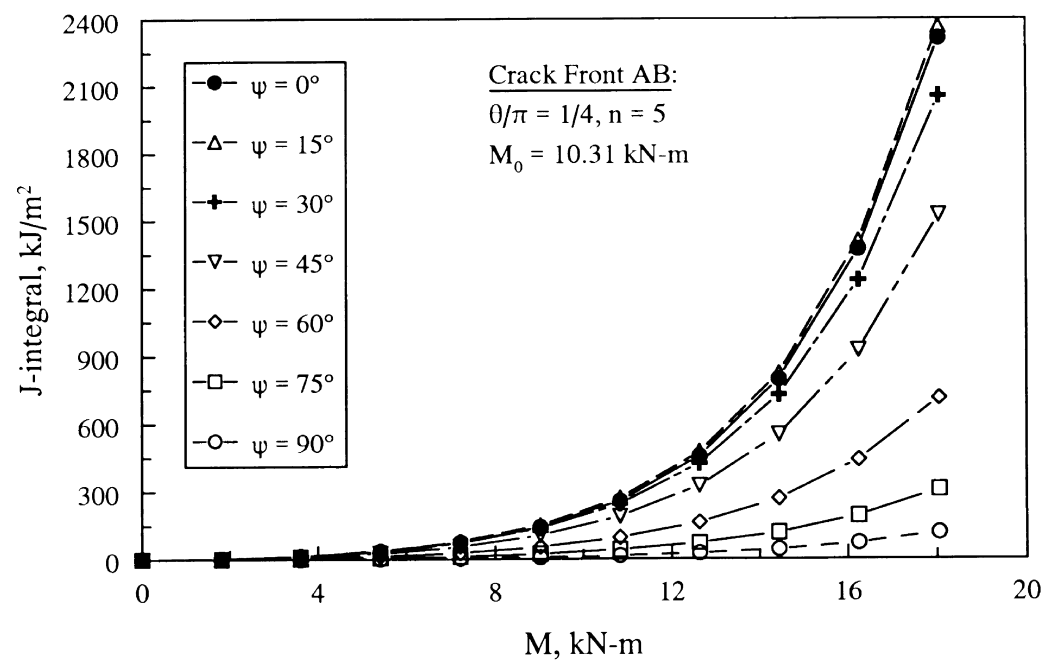

(c)

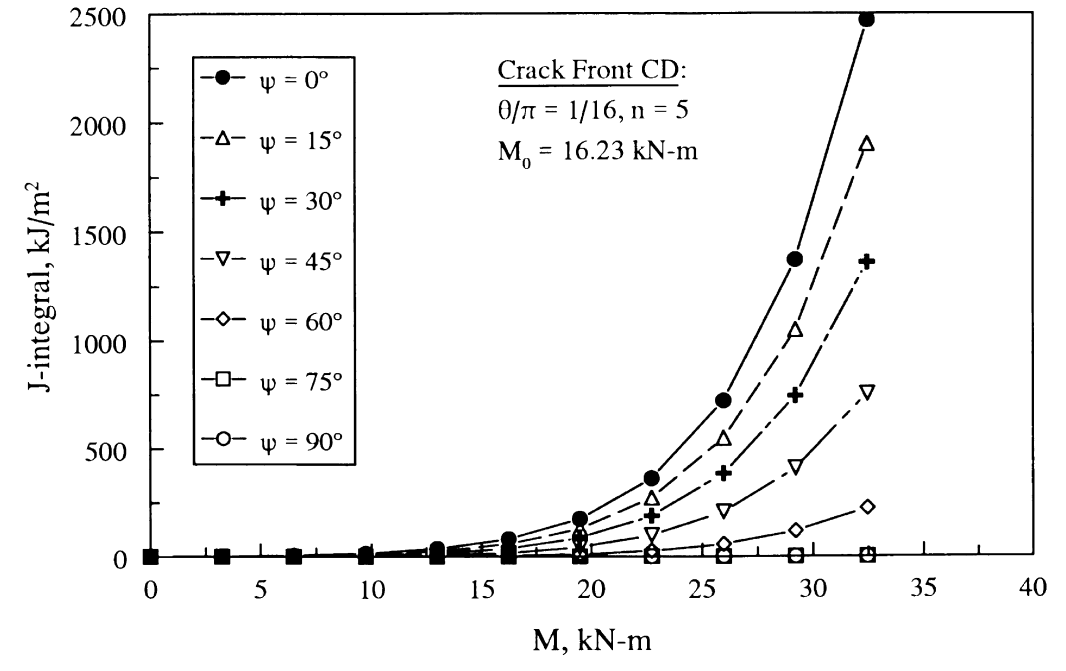

(b)

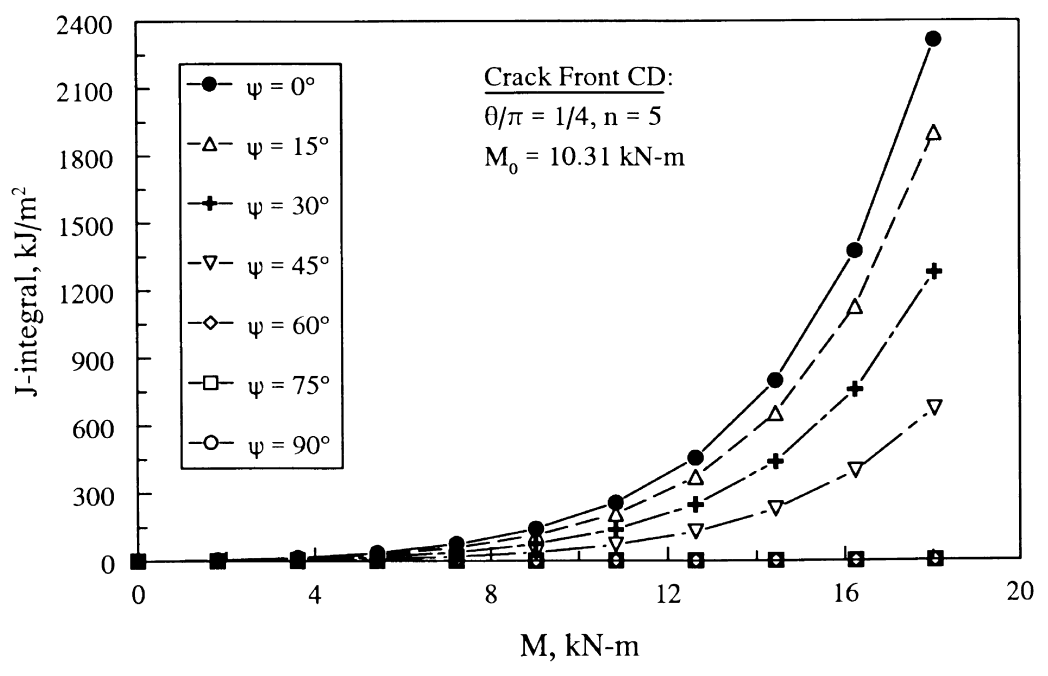

(d)

Fig. 2. $J$-integral for off-center cracks in pipe from past finite element analyses [7] (a) small crack $(\theta / \pi=1 / 16)$, crack front $\mathrm{AB} ;(\mathrm{b})$ small crack $(\theta / \pi=1 / 16)$, crack front $\mathrm{CD}$; (c) large crack $(\theta / \pi=1 / 14)$, crack front $A B$; (d) large crack $(\theta / \pi=1 / 14)$, crack front $C D$. 
and

$J_{\mathrm{p}, 0}=\frac{\alpha \sigma_{0}^{2}}{E} R_{\mathrm{m}} \theta\left(1-\frac{\theta}{\pi}\right) h_{1}\left(\frac{\theta}{\pi}, n, \frac{R_{\mathrm{m}}}{t}\right)\left(\frac{M}{M_{0}}\right)^{n+1}$

are the well-known GE/EPRI equations ${ }^{1}[9,10]$ for elastic and plastic components of $J$, respectively, for a symmetrically centered crack, i.e., when $\psi=0, F\left(\theta / \pi, R_{\mathrm{m}} / t\right)$ and $h_{1}\left(\theta / \pi, n, R_{\mathrm{m}} / t\right)$ are elastic and plastic influence functions for symmetrically centered cracks, $M_{0}$ is a reference moment [9,10], and $K_{e, \psi}$ and $K_{p, \psi}$ are constant elastic and plastic correction factors for off-center cracks, respectively. The evaluations of $F\left(\theta / \pi, R_{\mathrm{m}} / t\right)$ and $h_{1}\left(\theta / \pi, n, R_{\mathrm{m}} / t\right)$ are described in Appendix A.

By comparing Eqs. (2) and (3), it is easy to show that

$K_{\mathrm{e}, \psi}=\frac{J_{\mathrm{e}, \psi}}{J_{\mathrm{e}, 0}}$

$K_{\mathrm{p}, \psi}=\frac{J_{\mathrm{p}, \psi}}{J_{\mathrm{p}, 0}}$.

With this new proposed method, i.e. Eq. (3), the $J$-integral for off-center cracks can be easily calculated when these two correction factors are prescribed for a given off-center angle, $\psi$.

\subsection{Calculation of correction factors}

From Eqs. (6) and (7), it can be seen that the proposed correction factors are simply a ratio of the $J$ values for the off-centered crack to those of the symmetrically centered crack. Based on Eqs. (6) and (7) and past finite element results [7], Table 2 shows the results of $K_{e, \psi}$ and $K_{p, \psi}$ at cracks fronts $\mathrm{AB}$ and $\mathrm{CD}$ for a pipe with $R_{\mathrm{m}} / t=10$ and various combinations of $\theta / \pi, \psi$, and $n$. For an off-center angle of zero, the correction factors should have a value of one, as is shown in Table 2 . For the intermediate $(\theta / \pi=$ $1 / 8)$ and large $(\theta / \pi=1 / 4)$ cracks, it is shown that at the $15^{\circ}$ offset, the correction factor values are sometimes greater than unity for crack front AB. This agrees with the trend discussed in Rahman and Firmature [7], in which $J$ values at crack front $\mathrm{AB}$ with small offset angle are larger than the values obtained with a symmetrically centered crack. For all other cases the correction factors are less than 1.0, reflecting the fact that most of the time the centered crack is more critical than the off-centered crack. Correction factors close to zero were also found for offset angles that move the crack front $\mathrm{CD}$ below the bending axis and, therefore, may cause it to be closed.

\subsection{Response surface approximation of correction factors}

In order to eliminate the interpolation that is usually

\footnotetext{
${ }^{1}$ Note, the well-known GE/EPRI method, which constitutes Eqs. (4) and (5), is one of many $J$-estimation methods currently available for analyzing pipes with symmetrically-centered cracks. See Rahman et al. [4] and Brust et al. [11] for other $J$-estimation methods.
}

necessary when using tabulated data, it was decided to fit the correction factors listed in Table 2 with an analytical equation. The data in Table 2 show almost linear variation with respect to the crack length, but their variation with respect to the offset angle is slightly more complex. Accordingly, a response surface equation given by

$$
\begin{aligned}
K_{I, \psi} & =1+\left[a_{0}(n)+a_{1}(n)\left(\frac{\theta}{\pi}\right)\right] \psi \\
+ & {\left[a_{2}(n)+a_{3}(n)\left(\frac{\theta}{\pi}\right)\right] \psi^{2}+\left[a_{4}(n)+a_{5}(n)\left(\frac{\theta}{\pi}\right)\right] \psi^{3} } \\
+ & {\left[a_{6}(n)+a_{7}(n)\left(\frac{\theta}{\pi}\right)\right] \psi^{4} }
\end{aligned}
$$

is proposed, where $K_{I, \psi}$ is either $K_{\mathrm{e}, \psi}$ or $K_{\mathrm{p}, \psi}$ (i.e. the elastic or plastic correction factor for an off-centered crack), and $a_{i}(n), i=0,1, \ldots, 7$, are surface fit coefficients that depend on the material hardening parameter, $n$, for a pipe with a given $R_{\mathrm{m}} / t$. The coefficients, $a_{i}(n)$, can be further approximated by a fourth-order polynomial equation represented by

$a_{i}(n)=\sum_{j=0}^{4} D_{i j} n^{j}$

in which $D_{i j}(i=0,1, \ldots, 7$, and $j=0,1, \ldots, 4)$ are the polynomial coefficients that solely depend on the $R_{\mathrm{m}} / t$ ratio of the pipe. Following least-squares curve-fit of data in Table $2, D_{i j}$ were estimated and are given in Appendix B for a pipe with $R_{\mathrm{m}} / t=10$. Using these values of $D_{i j}$, Fig. 3(a) and (b) show the plots of $K_{\mathrm{e}, \psi}$ $(n=1)$ and $K_{\mathrm{p}, \psi}(n=5)$, respectively, as a function of $\theta / \pi$ and $\psi$ for a pipe with $R_{\mathrm{m}} / t=10$. From these plots, it appears that Eqs. (8) and (9) can accurately represent the data in Table 2. In fact, the square of correlation coefficient ( $R^{2}$ statistic) between the surface fit equations and the data was at least $99 \%$ for all cases considered in this study.

Note, the analytical approximation of $K_{I, \psi}$, represented by Eqs. (8) and (9), allows closed-form evaluation of $J$-integral for off-center cracks. This would significantly reduce computational effort in performing probabilistic analysis that are described in the following section and also for any future crack growth study.

\subsection{Limitations of response surface approximation}

Even with the very good agreement between the calculated correction factors and their surface fits, two very important limitations still exist. First, as mentioned in Rahman and Firmature [7], because the finite element meshes were only designed for $15^{\circ}$ increments, it is unknown how well the surface fits actually describe the behavior of crack front $\mathrm{AB}$ for offset angles lower 

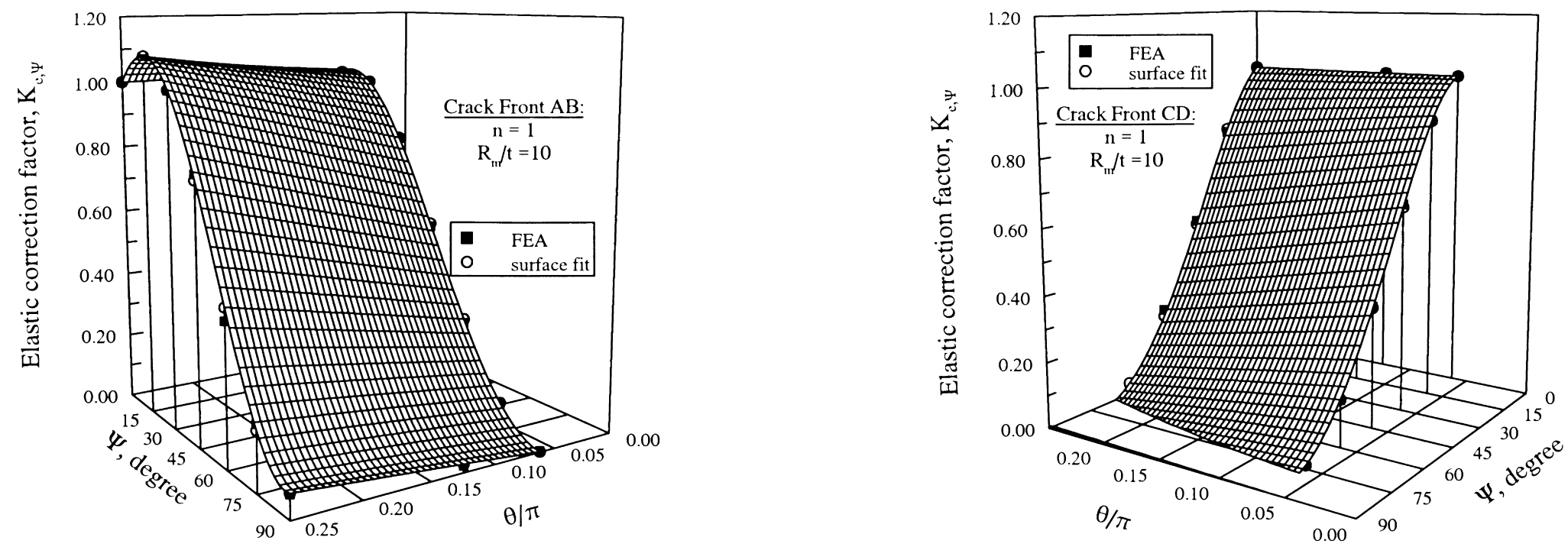

(a)
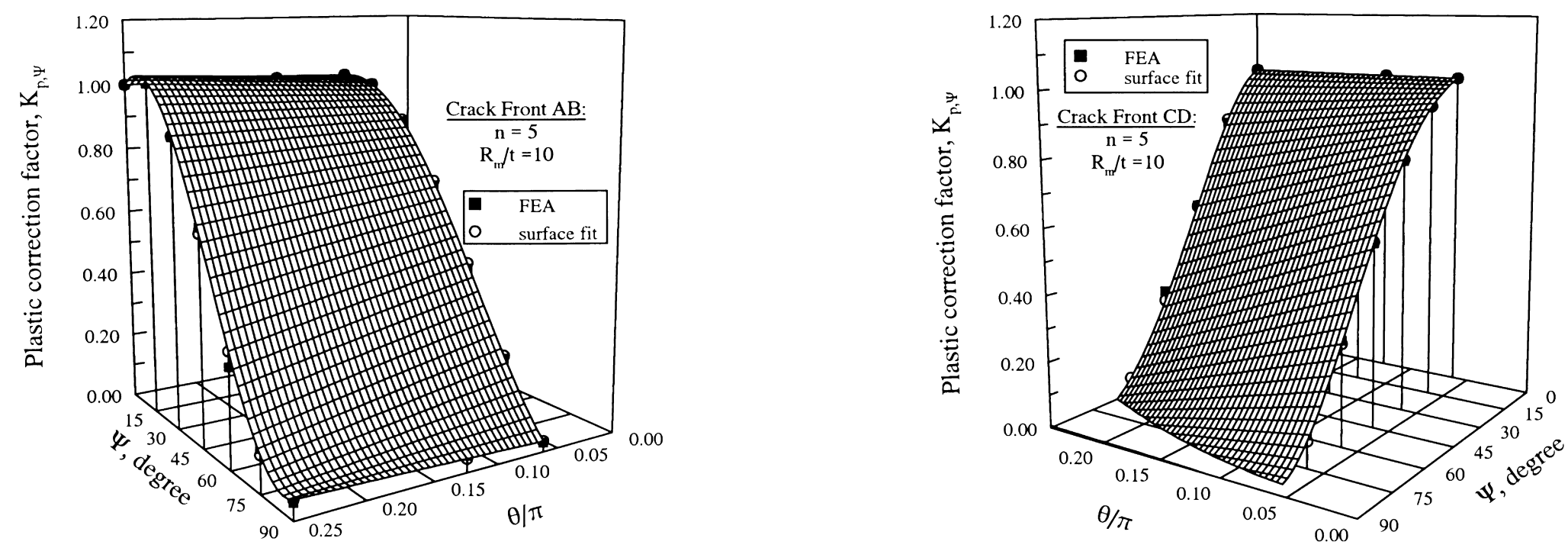

(b)

Fig. 3. Elastic and plastic correction factors for $R_{\mathrm{m}} / t=10$ (a) elastic correction factors for crack fronts $\mathrm{AB}$ and $\mathrm{CD}(n=1)$; (b) plastic correction factors for crack fronts $\mathrm{AB}$ and $\mathrm{CD}(n=5)$. 
Table 2

Elastic and plastic correction factors for $R_{\mathrm{m}} / t=10[\psi=0$ implies symmetrically centered crack; $n=1$ implies linear-elastic analysis $(\alpha=0)]$

\begin{tabular}{|c|c|c|c|c|c|c|c|}
\hline$n$ & $\psi=0^{\circ}$ & $\begin{array}{l}\psi=15^{\circ} \\
(\pi / 12 \mathrm{rad})\end{array}$ & $\begin{array}{l}\psi=30^{\circ} \\
(\pi / 6 \mathrm{rad})\end{array}$ & $\begin{array}{l}\psi=45^{\circ} \\
(\pi / 4 \mathrm{rad})\end{array}$ & $\begin{array}{l}\psi=60^{\circ} \\
(\pi / 3 \mathrm{rad})\end{array}$ & $\begin{array}{l}\psi=75^{\circ} \\
(5 \pi / 12 \mathrm{rad})\end{array}$ & $\begin{array}{l}\psi=90^{\circ} \\
(\pi / 2 \mathrm{rad})\end{array}$ \\
\hline \multicolumn{8}{|c|}{ Crack front $\mathrm{AB}[\theta / \pi=1 / 16]$} \\
\hline 1 & 1 & 0.974 & 0.807 & 0.552 & 0.287 & 0.087 & 0.004 \\
\hline 3 & 1 & 0.979 & 0.862 & 0.668 & 0.430 & 0.196 & 0.021 \\
\hline 5 & 1 & 0.980 & 0.870 & 0.685 & 0.455 & 0.222 & 0.027 \\
\hline 7 & 1 & 0.980 & 0.872 & 0.688 & 0.458 & 0.229 & 0.03 \\
\hline 10 & 1 & 0.980 & 0.867 & 0.677 & 0.447 & 0.224 & 0.03 \\
\hline \multicolumn{8}{|c|}{ Crack front $\mathrm{AB}[\theta / \pi=1 / 8]$} \\
\hline 1 & 1 & 1.016 & 0.882 & 0.640 & 0.364 & 0.125 & 0.020 \\
\hline 3 & 1 & 1.002 & 0.898 & 0.708 & 0.472 & 0.203 & 0.047 \\
\hline 5 & 1 & 0.993 & 0.882 & 0.689 & 0.458 & 0.198 & 0.048 \\
\hline 7 & 1 & 0.984 & 0.858 & 0.654 & 0.424 & 0.179 & 0.042 \\
\hline 10 & 1 & 0.973 & 0.821 & 0.596 & 0.366 & 0.145 & 0.032 \\
\hline \multicolumn{8}{|c|}{ Crack front $\mathrm{AB}[\theta / \pi=1 / 4]$} \\
\hline 1 & 1 & 1.079 & 0.998 & 0.784 & 0.418 & 0.190 & 0.068 \\
\hline 3 & 1 & 1.033 & 0.930 & 0.729 & 0.374 & 0.173 & 0.070 \\
\hline 5 & 1 & 1.014 & 0.872 & 0.639 & 0.291 & 0.124 & 0.048 \\
\hline 7 & 1 & 0.994 & 0.805 & 0.537 & 0.206 & 0.080 & 0.029 \\
\hline 10 & 1 & 0.970 & 0.724 & 0.431 & 0.138 & 0.048 & 0.016 \\
\hline \multicolumn{8}{|c|}{ Crack front $\operatorname{CD}[\theta / \pi=1 / 16]$} \\
\hline 1 & 1 & 0.875 & 0.641 & 0.370 & 0.141 & 0.015 & $-{ }^{\mathrm{a}}$ \\
\hline 3 & 1 & 0.920 & 0.752 & 0.525 & 0.278 & 0.071 & $-{ }^{\mathrm{a}}$ \\
\hline 5 & 1 & 0.929 & 0.775 & 0.557 & 0.312 & 0.094 & $-{ }^{\mathrm{a}}$ \\
\hline 7 & 1 & 0.934 & 0.783 & 0.568 & 0.323 & 0.108 & $-{ }^{\mathrm{a}}$ \\
\hline 10 & 1 & 0.936 & 0.783 & 0.564 & 0.322 & 0.117 & $-{ }^{\mathrm{a}}$ \\
\hline \multicolumn{8}{|c|}{ Crack front $\operatorname{CD}[\theta / \pi=1 / 8]$} \\
\hline 1 & 1 & 0.837 & 0.581 & 0.309 & 0.099 & $-^{\mathrm{a}}$ & $-{ }^{\mathrm{a}}$ \\
\hline 3 & 1 & 0.892 & 0.695 & 0.449 & 0.207 & $-{ }^{a}$ & $-{ }^{a}$ \\
\hline 5 & 1 & 0.896 & 0.703 & 0.460 & 0.222 & $-{ }^{a}$ & $-{ }^{\mathrm{a}}$ \\
\hline 7 & 1 & 0.892 & 0.691 & 0.445 & 0.215 & $-{ }^{\mathrm{a}}$ & $-{ }^{\mathrm{a}}$ \\
\hline 10 & 1 & 0.880 & 0.661 & 0.407 & 0.197 & $-{ }^{\mathrm{a}}$ & $-{ }^{\mathrm{a}}$ \\
\hline \multicolumn{8}{|c|}{ Crack front $\operatorname{CD}[\theta / \pi=1 / 4]$} \\
\hline 1 & 1 & 0.787 & 0.505 & 0.236 & $-^{\mathrm{a}}$ & $-^{\mathrm{a}}$ & $-^{\mathrm{a}}$ \\
\hline 3 & 1 & 0.833 & 0.582 & 0.322 & $-{ }^{\mathrm{a}}$ & $-^{\mathrm{a}}$ & $-{ }^{\mathrm{a}}$ \\
\hline 5 & 1 & 0.825 & 0.559 & 0.298 & $-{ }^{\mathrm{a}}$ & $-{ }^{\mathrm{a}}$ & $-{ }^{a}$ \\
\hline 7 & 1 & 0.812 & 0.520 & 0.258 & $-{ }^{a}$ & $-{ }^{a}$ & $-{ }^{a}$ \\
\hline 10 & 1 & 0.791 & 0.474 & 0.216 & $-{ }^{a}$ & $-{ }^{a}$ & $-{ }^{a}$ \\
\hline
\end{tabular}

${ }^{\mathrm{a}}$ Crack front closed.

than $15^{\circ}$. It was found that a threshold offset angle would exist below $15^{\circ}$ for each of the three crack lengths in this research; however, these threshold values were not determined in this study. It is expected that a small error will exist in this region due to a lack of data. For these reasons it is recommended that the surface fit equations for crack front $A B$ only be used for offset angles ranging from 15 to $90^{\circ}$ and only for crack lengths between $1 / 16$ and $1 / 4$ of the pipe circumference.

The second limitation is also related to the $15^{\circ}$ increment of the offset angle. Due to the set increment values and the varying crack lengths, the final data point for the two smaller cracks correspond to offset angles well before closure of crack front $\mathrm{CD}$. Because of this, the behavior of crack front $\mathrm{CD}$ is unknown from the last data point until closure for the two smaller cracks. However, for $\theta / \pi=1 / 4$ the final data point may fall at the largest offset for which front $\mathrm{CD}$ is not closed and hence, a discontinuity may occur at this point. Hence, the smaller cracks are expected to behave similarly in the same configuration. Due to this fact, the surface fit equations for crack front $\mathrm{CD}$ give reasonable approximations up to crack closure since they show the correct trend of a discontinuity at crack closure. However, since there are no data for the two smaller cracks at the point of crack closure, a region of extrapolation is needed between the final non-zero data points and the closure line [12]. The crack closure line is simply a straight line defining one edge of the extrapolation region in the $(\theta / \pi)-\psi$ plane. The crack is closed when $\psi+\theta>90^{\circ}$. If crack front CD is closed, then a value of zero should be used for the correction factor. Otherwise, correction factor data in this area should be used with caution since it is in an area of extrapolation. It is also recommended that the surface fit equations for crack front $\mathrm{CD}$ be used only for crack lengths between $1 / 16$ and 1/4 of the circumference. 


\section{Probabilistic fracture analysis}

\subsection{Random parameters and fracture response}

Consider a cracked structure with uncertain mechanical and geometric characteristics that is subject to random loads. Denote by $\mathbf{X}$ an $N$-dimensional random vector with components characterizing all uncertainties in the system and load parameters. For example, when an off-centered TWC pipe is considered, the possible random components are: crack geometric parameters, $\theta / \pi$ and $\psi$, material tensile parameters, $E, \nu, \alpha, n$, fracture toughness at crack initiation, $J_{\text {Ic }}$, and applied far-field moment, $M$. All or some of these variables can be modeled as random variables. Hence, any relevant fracture response, such as the $J$-integral, $J(\mathbf{X})$, should be evaluated by the probability

$F_{J}\left(j_{0}\right) \stackrel{\text { def }}{=} \operatorname{Pr}\left[J(\mathbf{X})<j_{0}\right]=\int_{J(\mathbf{X})<j_{0}} f_{\mathbf{X}}(\mathbf{x}) \mathrm{d} \mathbf{x}$

or the probability density function (PDF), $f_{J}\left(j_{0}\right)=$ $\mathrm{d} F_{J}\left(j_{0}\right) / \mathrm{d} j_{0}$, where $F_{J}\left(j_{0}\right)$ is the cumulative distribution function of $J$ and $f_{\mathbf{X}}(\mathbf{x})$ is the known joint PDF of input random vector $\mathbf{X}$.

The fracture parameter $J$ can also be applied to calculate structural integrity and hence, failure probability, $P_{\mathrm{F}}$, of cracked structures. This failure probability depends on the failure criterion. For example, if the initiation of crack growth in the TWC pipe constitutes a failure condition, $P_{\mathrm{F}}$ can be written as

$P_{\mathrm{F}}=\operatorname{Pr}[g(\mathbf{X})<0]=\int_{g(x)<0} f_{\mathbf{X}}(\mathbf{x}) \mathrm{d} \mathbf{x}$

where

$g(\mathbf{X})=J_{\mathrm{Ic}}-J(\mathbf{X})$.

Note, if $j_{0}$ represents the material fracture toughness at crack initiation $\left(J_{\mathrm{Ic}}\right), P_{\mathrm{F}}$ is simply the complement of $F_{J}\left(j_{0}\right)$, i.e., $P_{\mathrm{F}}=1-F_{J}\left(j_{0}\right)$. The failure probability, $P_{\mathrm{F}}$ in Eq. (11) corresponds to the probability of initiation of crack growth, which provides a conservative estimate of structural performance. A more realistic evaluation of structural reliability requires calculating the probability of fracture instability following crack initiation. See Ref. [13] for further details.

\subsection{Structural reliability analysis}

The generic expression for the probabilities in Eqs. (10) and (11) involves multi-dimensional probability integration for its evaluation. Standard reliability methods, such as firstand second-order reliability methods (FORM/SORM) [14-18], Monte Carlo simulation (MCS) [19], and Monte Carlo with Importance Sampling (MCIS) [20-22], can be used to compute these probabilities. In this study, FORM/SORM, MCS, and MCIS were used to calculate the probability of failure, $P_{\mathrm{F}}$ in Eq. (11) assuming a generic $N$-dimensional random vector $\mathbf{X}$ and the perfor- mance function $g(\mathbf{x})$ defined by Eq. (12). For brevity, only the results of FORM/SORM will be presented in this paper. A brief description of FORM/SORM is given in the following subsection.

First- and Second-Order Reliability Methods: First- and second-order reliability methods are based on linear (firstorder) and quadratic (second-order) approximations of the limit state surface $g(\mathbf{x})=0$ tangent to the closest point of the surface to the origin of the space. The determination of this point involves nonlinear constrained optimization and is performed in the standard Gaussian image of the original space. The FORM/SORM algorithms involve several steps. First, the space $\mathbf{x}$ of original random vector $\mathbf{X}$ is transformed into a new $\mathrm{N}$-dimensional space $\mathbf{u}$ consisting of independent standard Gaussian vector $\mathbf{U}$. The original limit state $g(\mathbf{x})=$ 0 then becomes mapped into the new limit state $g_{\mathrm{U}}(\mathbf{u})=0$ in the $\mathbf{u}$ space. Second, the point on the limit state $g_{\mathrm{U}}(\mathbf{u})=0$ having the shortest distance to the origin of the $\mathbf{u}$ space is determined by using an appropriate nonlinear optimization algorithm. This point is referred to as the design point or most probable point, and has a distance $\beta_{\mathrm{HL}}$ (known as the reliability index) to the origin of the $\mathbf{u}$ space. Third, the limit state $g_{\mathrm{U}}(\mathbf{u})=0$ is approximated by a surface tangent to it at the design point. Let such limit states be $g_{\mathrm{L}}(\mathbf{u})=0$ and $g_{\mathrm{Q}}(\mathbf{u})=0$, which correspond to approximating surfaces as hyperplane (linear or first-order) and hyperparaboloid (quadratic or second-order), respectively. The probability of failure $P_{\mathrm{F}}$ [Eq. (11)] is thus approximated by $\operatorname{Pr}\left[g_{\mathrm{L}}(\mathbf{u})<\right.$ $0]$ in FORM and $\operatorname{Pr}\left[g_{\mathrm{Q}}(\mathbf{u})<0\right]$ in SORM. These first-order and second-order estimates $P_{\mathrm{F}, 1}$ and $P_{\mathrm{F}, 2}$ are given by [14-18,22]

$P_{\mathrm{F}, 1}=\Phi\left(-\beta_{\mathrm{HL}}\right)$

$P_{\mathrm{F}, 2} \cong \Phi\left(-\beta_{\mathrm{HL}}\right) \prod_{i=1}^{N-1}\left(1-\kappa_{i} \frac{\phi\left(-\beta_{\mathrm{HL}}\right)}{\Phi\left(-\beta_{\mathrm{HL}}\right)}\right)^{-1 / 2}$

where

$\phi(u)=\frac{1}{\sqrt{2 \pi}} \exp \left(-\frac{1}{2} u^{2}\right)$

and

$\Phi(u)=\frac{1}{\sqrt{2 \pi}} \int_{-\infty}^{u} \exp \left(-\frac{1}{2} \xi^{2}\right) \mathrm{d} \xi$

are the probability density and cumulative distribution functions, respectively, of a standard Gaussian random variable, and $\kappa_{\mathrm{i}}$ 's are the principal curvatures of the limit state surface at the design point. FORM/SORM are analytical probability computation methods. Each input random variable and the performance function $g(\mathbf{x})$ must be continuous. Depending on the solver for nonlinear programming, an additional requirement regarding smoothness, i.e. the differentiability of $g(\mathbf{x})$ may be required. In this study, a sequential quadratic programming method with an appropriate tolerance criterion for the convergence was used to solve the 
Table 3

Statistical properties of random input

\begin{tabular}{|c|c|c|c|c|}
\hline Random variable & Mean & $\mathrm{COV}^{\mathrm{a}}$ & Probability distribution & Reference \\
\hline Elastic modulus $(E)$ & $182.7 \mathrm{GPa}$ & 0.05 & Gaussian & {$[23]$} \\
\hline Ramberg-Osgood coefficient $(\alpha)$ & 8.073 & 0.439 & Lognormal & {$[23]$} \\
\hline Ramberg-Osgood exponent $(n)$ & 3.8 & 0.146 & Lognormal & {$[23]$} \\
\hline Initiation fracture toughness $\left(J_{\text {Ic }}\right)$ & $1,242.6 \mathrm{~kJ} / \mathrm{m}^{2}$ & 0.47 & Lognormal & {$[23]$} \\
\hline Normalized crack angle $(\theta / \pi)$ & $1 / 8$ & 0.1 & Lognormal & $-{ }^{b}$ \\
\hline Bending moment $(M)$ & Variable $^{c}$ & 0.1 & Gaussian & $-{ }^{b}$ \\
\hline
\end{tabular}

${ }^{\mathrm{a}}$ Coefficient of variation $(\mathrm{COV})=$ standard deviation $/$ mean

b Arbitrarily assumed.

c Arbitrarily varied.

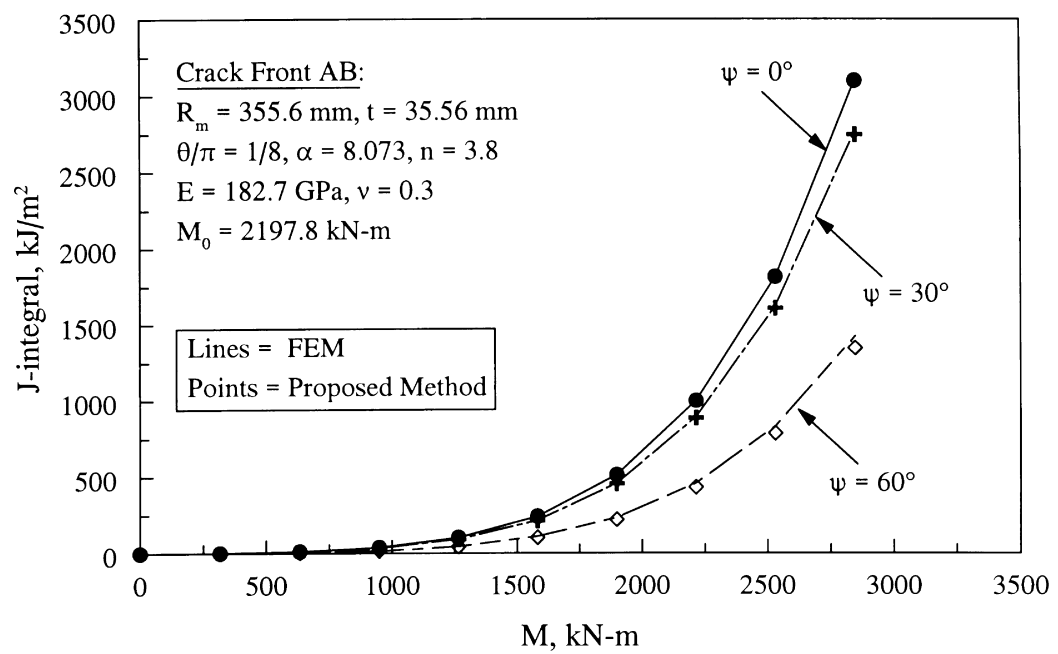

(a)

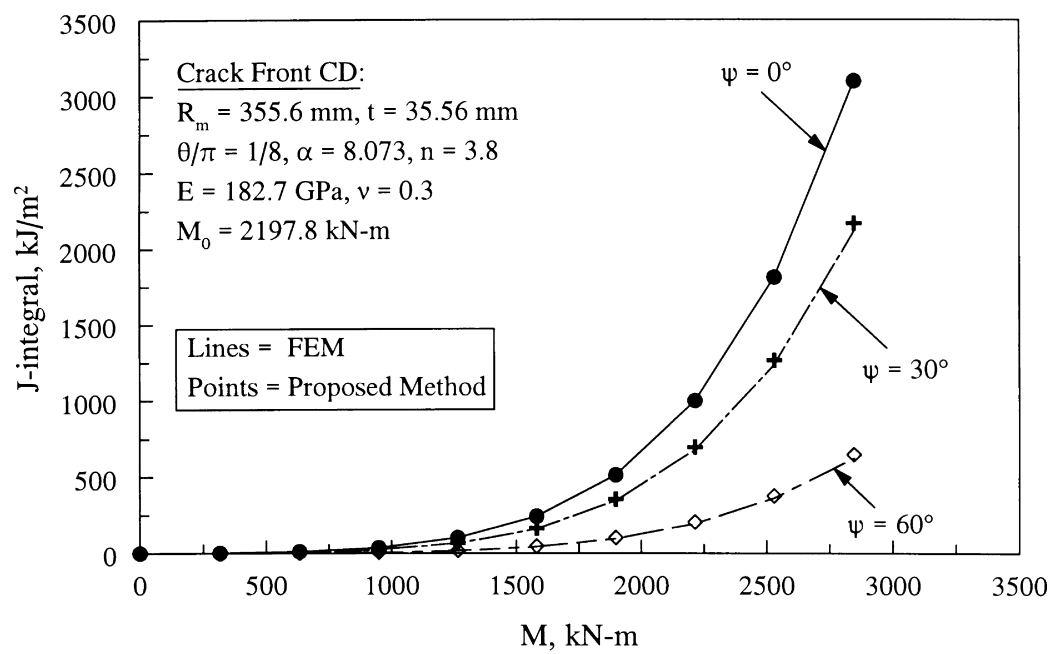

(b)

Fig. 4. Comparisons of predicted $J$ by FEM and proposed estimation method (a) crack front AB; (b) crack front CD. 


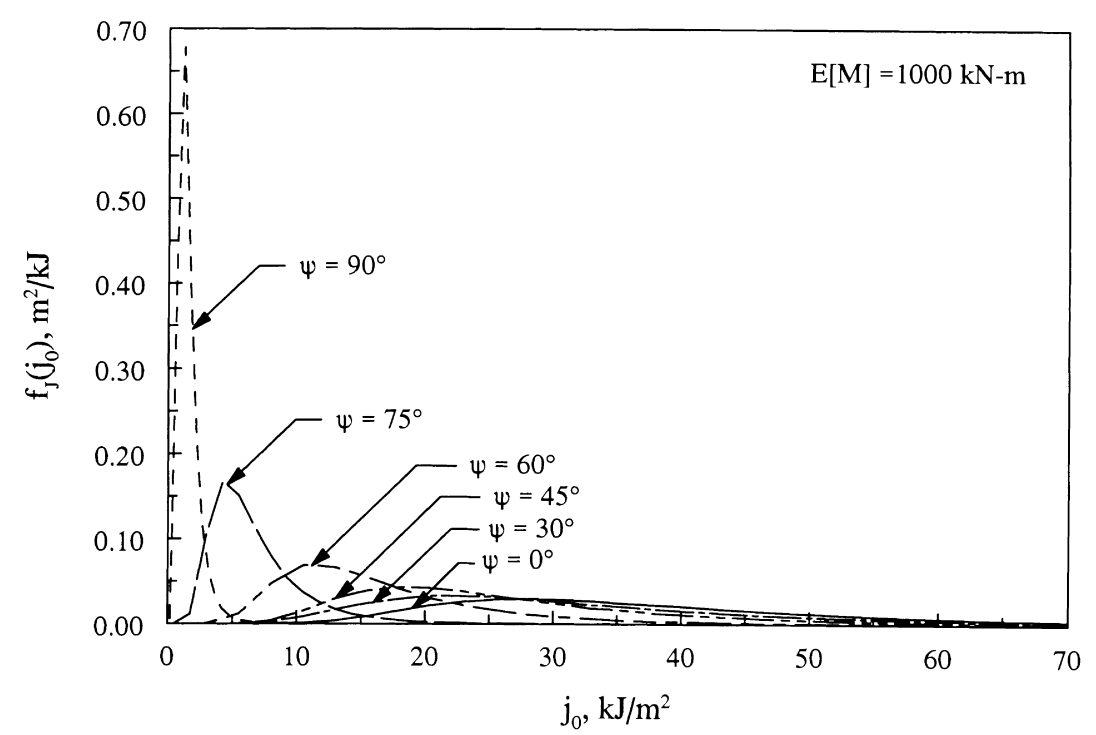

Fig. 5. Probability densities of $J$ for various off-center cracks in pipes.

optimization problem. Further details of FORM/SORM equations are available in Refs. [14-18].

\section{Numerical example}

Consider a pipe with a circumferential through-wall crack subjected to remote bending moment, $M$. The crack is offcentered by an angle, $\psi$ with respect to the bending plane. The pipe has a mean radius, $R_{\mathrm{m}}=355.6 \mathrm{~mm}$ (14 in), a wall thickness, $t=35.56 \mathrm{~mm}$ (1.4 in), and a normalized crack angle, $\theta / \pi$. The pipe is made of TP304 stainless steel with an operating temperature of $288^{\circ} \mathrm{C}\left(550^{\circ} \mathrm{F}\right)$. The pipe geometry is shown in Fig. 1(a) and (b).

Table 3 shows the means, coefficients of variation, and probability distributions of tensile parameters $(E, \alpha, n)$, fracture toughness at crack initiation $\left(J_{\text {Ic }}\right)$, normalized crack angle $(\theta / \pi)$, and bending moment $(M)$. The off-center angle $(\psi)$ was treated as both a deterministic and a random variable. The statistics in Table 3 came from statistical characterization of actual material property data [23]. These random variables were assumed to be statistically independent. In addition, the following deterministic values were used: $\sigma_{0}=154.78 \mathrm{MPa}(22,450 \mathrm{psi})$ and $\nu=0.3$.

\subsection{Deterministic results}

Fig. 4(a) and (b) show the deterministic comparisons of predicted $J$ for $\psi=0,30$ and $60^{\circ}$ by the proposed method [Eq. (3)] and elastic-plastic finite element results at crack fronts $\mathrm{AB}$ and $\mathrm{CD}$, respectively. The mean values of random input given in Table 3 and other deterministic parameters described above were used to conduct these analyses. In Fig. 4(a) and (b), the solid points indicate the $J$ values from the

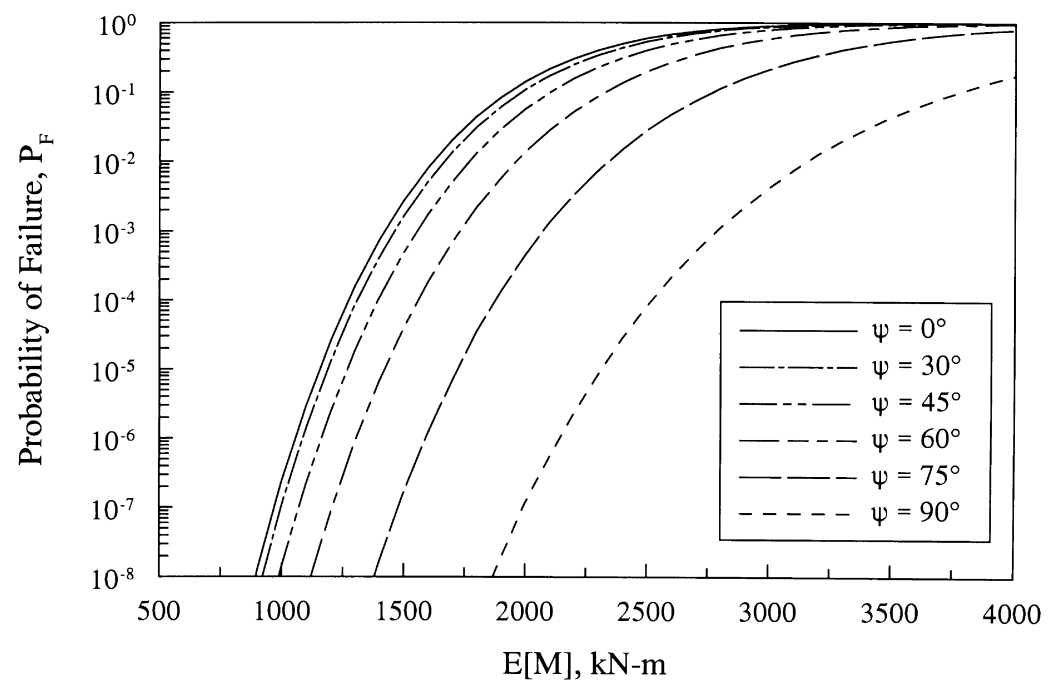

Fig. 6. Probability of failure for various off-center cracks in pipes. 


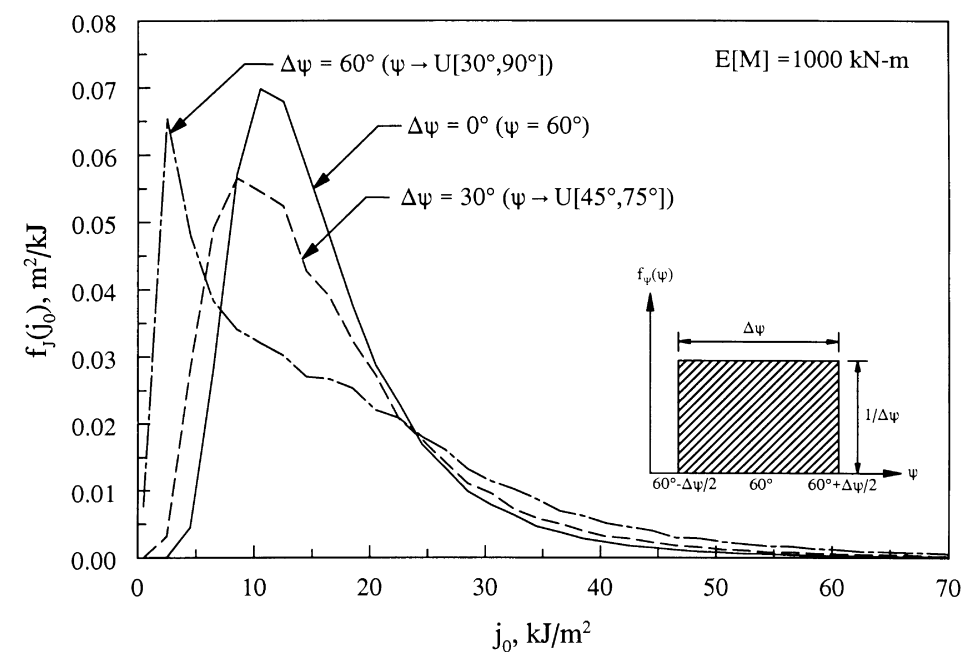

Fig. 7. Probability densities of $J$ for uncertain off-center cracks in pipes.

proposed estimation method and the lines represent the $J$ solutions from finite element analysis using the ABAQUS code. The predicted values of $J$ from Eq. (3) compare very well with the generally more accurate finite element results. This also verifies the adequacy of proposed analytical equations for elastic-plastic analysis of off-center cracks in pipes.

\subsection{Probabilistic results}

The second-order reliability method was applied to determine the probabilistic characteristics of $J$ for offcentered cracked pipes subject to pure bending. Since $J$ at crack front $\mathrm{AB}$ is always larger than $J$ at crack front $\mathrm{CD}$, all following analysis and results are based on $J$ at crack front AB. Fig. 5 shows the computed probability densities of $J\left[f_{J}\left(j_{0}\right)\right]$ for off-center angles, $\psi=0,30,45,60,75$, and $90^{\circ}$ when $E[M]=1000 \mathrm{kN} \mathrm{m}$ (8851 kip in), where $E[\cdot]$ is the mean operator. They were obtained by repeated SORM analysis for various thresholds of $J$, i.e. by calculating the probability in Eq. (10) as a function of $j_{0}$ and then taking numerical derivative of this probability with respect to $j_{0}$. As expected, the probability mass shifts from right to left when $\psi$ increases. This is consistent with deterministic observations in Figs. 2 and 4.

Fig. 6 shows the plots of $P_{\mathrm{F}}$ vs. $E[M]$ for $\psi=0,30,45$, 60,75 and $90^{\circ}$ obtained by SORM. They all consistently indicate that $P_{\mathrm{F}}$ increases as $E[M]$ increases, and it approaches unity when $E[M]$ becomes very large. For a given applied moment, the failure probability decreases when $\psi$ increases. This is consistent with the fact that the $J$-integral for an off-center crack is generally lower than that for a symmetrically centered crack. In the past, the probabilistic analysis of an off-center crack in pipes involved simplifying the crack to be symmetrically centered with respect to the bending plane of the pipe [24]. This study

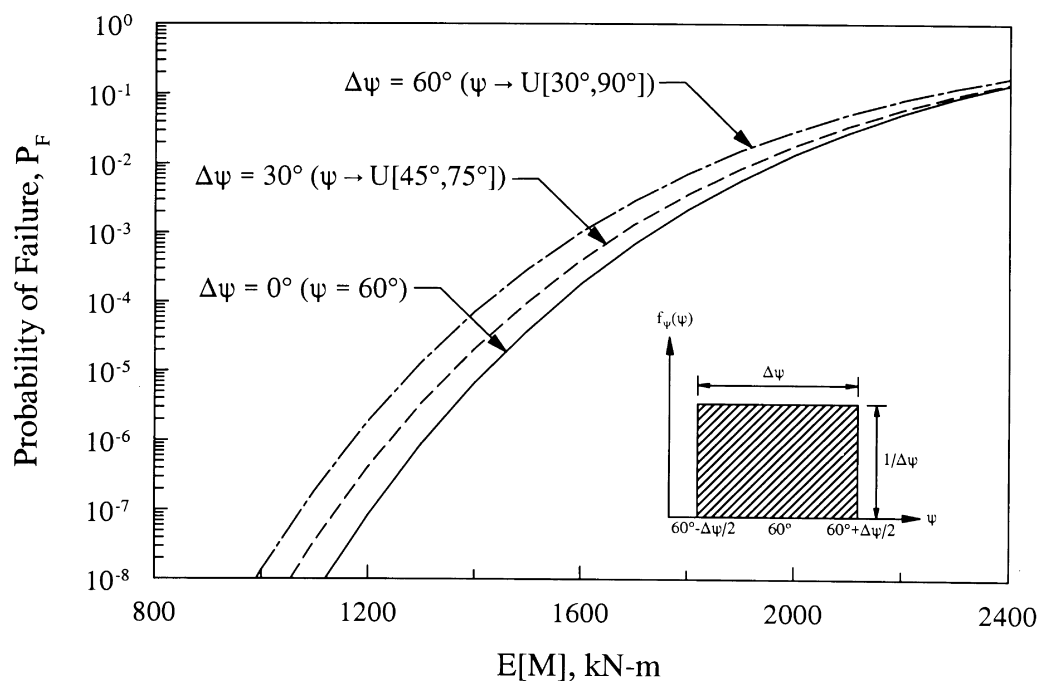

Fig. 8. Probability of failure for uncertain off-center cracks in pipes. 


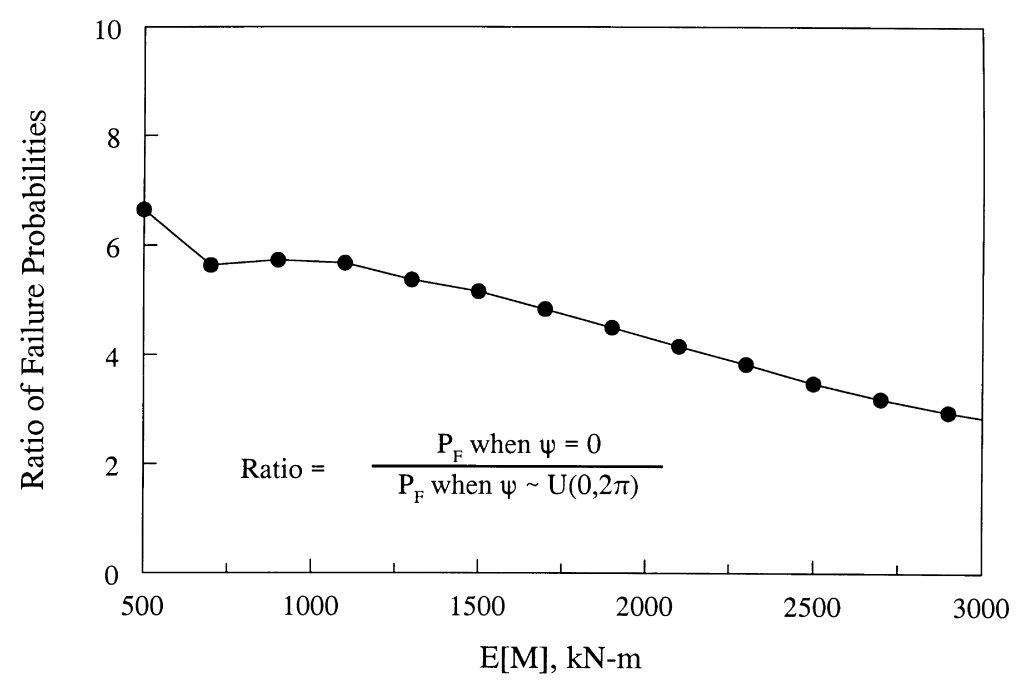

Fig. 9. Ratio of failure probabilities vs. applied moment.

provides a quantitative framework for analyzing off-centercracked pipes without having to make such unnecessary assumption or to perform expensive elastic-plastic finite element analyses. This is obviously due to the proposed method that is capable of predicting $J$ for off-centered cracks in pipes by simple analytical equations. Note, the SORM probabilities presented here were also validated against the results of MCS and MCIS methods. For brevity, they were not presented here. See previous work of authors on the validation of FORM/SORM results of symmetrically centered cracks by the simulation methods $[13,23]$.

The results presented so far [see Figs. 5 and 6] are valid for only deterministic values of the off-center angle. In reality, this off-center angle is highly likely to be a random variable. As an example, assume that this off-center angle, $\psi$, is uniformly distributed over $\left[60^{\circ}-\Delta \psi / 2,60^{\circ}+\Delta \psi / 2\right]$, where $\Delta \psi$ represents the spread of its uniform probability density function. With this uniform distribution of $\psi$ and other input properties described earlier, Figs. 7 and 8 show the plots of SORM-generated probability densities of $J$ and failure probability, respectively, when $\Delta \psi=0 \quad(\psi$ is deterministic), $30^{\circ}\left(\psi\right.$ is uniform over $\left[45^{\circ}, 75^{\circ}\right]$ ), and $60^{\circ}$ ( $\psi$ is uniform over $\left[30^{\circ}, 90^{\circ}\right]$ ). These results show that the uncertainty in $\psi$ can have a significant effect on the probabilistic characteristics of $J$ and failure probability, particularly when $\Delta \psi$ is large. When $\Delta \psi$ is $30^{\circ}$ (small uncertainty), the failure probability curve is close to the results of $\psi=60^{\circ}$, which is the mean value of $\psi$. However, when $\Delta \psi$ is $60^{\circ}$ (large uncertainty), the failure probabilities can be much higher than those for $\psi=60^{\circ}$ and hence, its uncertainty should be accounted for probabilistic pipe fracture evaluations. While these trends are somewhat expected, the above analyses provide a quantitative measure of these failure probabilities.

Finally, Fig. 9 shows a plot of the ratio of two failure probabilities - one calculated when $\psi=0$, and the other calculated when $\psi$ is uniformly distributed over $\left[0,360^{\circ}\right]$ - as a function of the mean applied moment. This ratio quantifies the factor by which the failure probability estimate assuming a worst-case condition (i.e. when $\psi=0$ ) overestimates the failure probability when $\psi$ is random and equally likely to take on an angle between 0 and $360^{\circ}$. The results in Fig. 9 indicate that the ratio or the factor varies from 2 to 7 depending on the bending moment applied. All probabilities defined above were calculated by SORM.

\section{Summary and conclusions}

A probabilistic methodology was developed for fracturemechanics analysis of off-center cracks in pipes subject to pure bending moment. It is based on: (1) analytical approximation of $J$-integral; (2) statistical models of uncertainties in loads, material properties, and crack geometry; and (3) standard computational methods of structural reliability theory. The results from 105 deterministic finite element analysis, performed for a wide variety of crack sizes, offset crack angles, and material hardening parameter, were used to develop elastic and plastic correction factors for predicting $J$-integral. Following response surface approximations of these correction factors, new closed-form analytical equations were developed to calculate $J$ for a given pipe geometry, crack size, off-center angle, and material properties.

The analytical equations were subsequently applied for probabilistic fracture-mechanics analysis. The second-order reliability method was used to determine the probabilistic characteristics of the $J$-integral. The same method was used later to predict the failure probability based on the initiation of crack growth. A numerical example is presented to illustrate the proposed methodology. The results show that:

- The probabilistic characteristics of $J$ and failure probability are strongly dependent on the off-center 
crack angle. For a given applied moment, the failure probability of a pipe with an off-center crack is generally lower than that of a pipe with symmetrically centered crack. Hence, simplifying an off-center crack to be symmetrically centered crack can produce significant conservatism in predicting failure probabilities.

- A large uncertainty in the off-center crack angle, if exists, can have a significant effect on the failure probability of pipes. For example, when the off-center crack angle is uniformly distributed over 30 and $90^{\circ}$, the failure probability can be a order of magnitude larger than that calculated when the off-center angle is assigned its mean value.

- The ratio of failure probabilities based on worst-case condition (symmetrically centered crack) to those obtained from random off-center crack angle that is uniformly distributed over [0 and $360^{\circ}$ ] varies between 2 and 7, depending on the bending moment applied.

The proposed analytical equations of $J$ for off-center cracks in pipes can also be potentially used for crack-growth studies. From crack-growth analysis, one can predict fracture instability and corresponding probability with little effort. These are subjects of current research by the authors.

\section{Acknowledgements}

The authors would like to acknowledge the support by the US National Science Foundation (Grant No. CMS9833058).

\section{Appendix A. Influence functions for symmetric cracks}

In Eq. (4), $F\left(\theta / \pi, R_{\mathrm{m}} / t\right)$ is a dimensionless elastic influence function that depends on pipe and crack geometry. According to Rahman [13]:

$$
\begin{aligned}
F\left(\theta / \pi, R_{\mathrm{m}} / t\right)= & 1+\left\{\begin{array}{lll}
A_{1} & A_{2} & A_{3}
\end{array}\right\}\left\{\begin{array}{c}
(\theta / \pi)^{1.5} \\
(\theta / \pi)^{2.5} \\
(\theta / \pi)^{3.5}
\end{array}\right\} \\
& \times\left\{\begin{array}{llll}
B_{1} & B_{2} & B_{3} & B_{4}
\end{array}\right\}\left\{\begin{array}{c}
1 \\
\left(R_{\mathrm{m}} / t\right) \\
\left(R_{\mathrm{m}} / t\right)^{2} \\
\left(R_{\mathrm{m}} / t\right)^{3}
\end{array}\right\}
\end{aligned}
$$

where $A_{i}(i=1-3)$ and $B_{i}(i=1-4)$ are constant coefficients. Let $\mathbf{A}=\left\{\begin{array}{lll}A_{1} & A_{2} & A_{3}\end{array}\right\}^{\mathrm{T}} \quad$ and $\quad \mathbf{B}=$ $\left\{\begin{array}{llll}B_{1} & B_{2} & B_{3} & B_{4}\end{array}\right\}^{\mathrm{T}}$ be two real vectors with the coefficients, $A_{i}$ and $B_{i}$ as their components, respectively. Using best fit of finite element results, $\mathbf{A}$ and $\mathbf{B}$ are given by [13]:

$$
\begin{aligned}
& \mathbf{A}=\left\{\begin{array}{llll}
0.006215 & 0.013304 & -0.01838
\end{array}\right\}^{\mathrm{T}} \\
& \mathbf{B}=\left\{\begin{array}{llll}
175.577 & 91.69105 & -5.53806 & 0.15116
\end{array}\right\}^{\mathrm{T}}
\end{aligned}
$$

In Eq. (5), $h_{1}\left(\theta / \pi, n, R_{\mathrm{m}} / t\right)$ is a dimensionless plastic influence function that depends on pipe geometry, crack geometry, and material hardening exponent. According to Rahman [13],

$$
\begin{aligned}
h_{1}\left(\theta / \pi, n, R_{\mathrm{m}} / t\right)= & \left\{\begin{array}{llll}
1 & (\theta / \pi) & (\theta / \pi)^{2} & (\theta / \pi)^{3}
\end{array}\right\} \\
& \times\left[\begin{array}{cccc}
C_{00} & C_{10} & C_{20} & C_{30} \\
C_{01} & C_{11} & C_{21} & C_{31} \\
C_{02} & C_{12} & C_{22} & C_{32} \\
C_{03} & C_{13} & C_{23} & C_{33}
\end{array}\right]\left\{\begin{array}{c}
1 \\
n \\
n^{2} \\
n^{3}
\end{array}\right\}
\end{aligned}
$$

where $C_{i j}(i, j=0-3)$ are coefficients which depend on $R_{\mathrm{m}} / t$ and can also be calculated from best fit of finite element results [13]. Let $C=\left[C_{i j}\right], i, j=0-3$, be a real matrix with the coefficients, $C_{i j}$ as its components. According to Rahman [13], $\mathbf{C}$ is given by:

For $R_{\mathrm{m}} / t=5$,

$\mathbf{C}=\left[\begin{array}{rrrr}3.74009 & 1.43304 & -0.10216 & 0.0023 \\ -0.19759 & -10.19727 & -0.45312 & 0.04989 \\ 36.42507 & 17.03413 & 3.36981 & -0.21056 \\ -70.4846 & -14.69269 & -2.90231 & 0.15165\end{array}\right]$

For $R_{\mathrm{m}} / t=10$,

$\mathbf{C}=\left[\begin{array}{rrrr}3.39797 & 1.31474 & -0.07898 & 0.00287 \\ -3.07265 & 4.34242 & -2.48397 & 0.11476 \\ 131.7381 & -79.02833 & 16.18829 & -0.66912 \\ -234.6221 & 117.0509 & -20.30173 & 0.79506\end{array}\right]$

For $R_{\mathrm{m}} / t=20$

$\mathbf{C}=\left[\begin{array}{rrrc}4.07828 & -1.55095 & 0.67206 & -0.0442 \\ -18.21195 & 69.92277 & -18.41884 & 1.11308 \\ 357.4929 & -453.1582 & 108.0204 & -6.56651 \\ -602.7576 & 617.9074 & -144.9435 & 8.9022\end{array}\right]$

See Rahman [13] for further explanations on how these coefficients were calculated.

\section{Appendix B. Coefficients $D_{i j}$}

Let $\mathbf{D}=\left[D_{i j}\right], i=0,1, \ldots, 7$, and $j=0,1, \ldots, 4$, be a real matrix with the coefficients, $D_{i j}$, as its components. Following 
least-squares curve fit, $\mathbf{D}$ for $R_{\mathrm{m}} / t=10$ is given by:

At crack front $\mathrm{AB}$,

$\mathbf{D}=\left[\begin{array}{rrrrr}0.3528 & -0.2522 & 0.0631 & -0.0074 & 0.0003 \\ 2.7962 & -1.0617 & 0.2940 & -0.0266 & 0.0008 \\ -2.9981 & 1.6897 & -0.4233 & 0.0496 & -0.0021 \\ 3.7670 & -2.0229 & 0.3196 & -0.0762 & 0.0047 \\ 2.0620 & -1.4627 & 0.3823 & -0.0467 & 0.0020 \\ -7.1406 & 2.5393 & -0.5405 & 0.1119 & -0.0064 \\ -0.3596 & 0.3150 & -0.0885 & 0.0115 & -0.0005 \\ 2.3539 & -0.5121 & 0.1318 & -0.0327 & 0.0019\end{array}\right]$

At crack front CD,

$\mathbf{D}=\left[\begin{array}{rrrrr}0.1444 & -0.2620 & 0.0651 & -0.0064 & 0.0002 \\ -3.1782 & 2.5246 & -0.6779 & 0.0734 & -0.0029 \\ -3.3451 & 2.3779 & -0.5637 & 0.0583 & -0.0022 \\ 4.2744 & -8.1376 & 1.9887 & -0.2243 & 0.0094 \\ 2.9435 & -2.6898 & 0.6399 & -0.0670 & 0.0026 \\ -0.6972 & 6.2504 & -1.4695 & 0.1750 & -0.0077 \\ -0.7191 & 0.8152 & -0.1955 & 0.0207 & -0.0008 \\ -0.4701 & -1.3246 & 0.3025 & -0.0393 & 0.0019\end{array}\right]$

\section{References}

[1] USNRC, Report to the US Nuclear Regulatory Commission Piping Review Committee. Prepared by Pipe Break Task Group, NUREG/ CR-1061, Vol. 3, US Nuclear Regulatory Commission, Washington, DC, November 1984.

[2] Federal Register, Standard Review Plan, Section 3.6.3. Leak-beforebreak evaluation procedures. Public comment paper, Vol. 52, No. 167, Notices, p. 32 626-32 633, August 1987.

[3] American Society of Mechanical Engineers. ASME Boiler \& Pressure Vessel Code, Section XI, 1989.

[4] Rahman S, Brust F, Ghadiali N, Choi YH, Krishnaswamy P, Moberg F, Brickstad B, Wilkowski G. Refinement and evaluation of crackopening-area analyses for circumferential through-wall cracks in pipes. NUREG/CR-6300, US Nuclear Regulatory Commission, Washington, DC, April 1995.

[5] Rahman S, Ghadiali N, Wilkowski G, Bonora N. Effects of offcentered crack and restraint of induced bending on the crackopening-area analysis of pipes. In: Mehta $\mathrm{H}$, editor. Proceedings of 1995 ASME/JSME Pressure Vessels and Piping Conference, Honolulu, Hawaii, Fatigue and Fracture Mechanics in Pressure Vessels and Piping, PVP, 1995;304:149-62.

[6] Rahman S, Ghadiali N, Wilkowski G, Bonora N. Effects of off-centered crack and restraint of induced bending due to pressure on the crack-opening-area analysis of pipes. Nuclear Engineering and Design 1997;167:55-67.

[7] Rahman S, Firmature R. Elastic plastic analysis of off-center cracks in cylindrical structures. In: Rahman S, editor. Proceedings of 1998 ASME/JSME Pressure Vessels and Piping Conference, San Diego, CA, Fatigue, Fracture, and Residual Stresses, PVP, 1998;373:489-509.

[8] ABAQUS, User's Guide and Theoretical Manual, Version 5.6. Hibbitt, Karlsson, \& Sorensen, Inc., Pawtucket, RI, 1997.

[9] Kumar V, German MD, Wilkening WW, Andrews WR, de Lorenzi HG, Mowbray DF. Advances in ElasticPlastic Fracture Analysis, EPRI NP3607, Electric Power Research Institute, Palo Alto, CA, 1984.

[10] Kumar V, German M. Elastic-plastic fracture analysis of throughwall and surface flaws in cylinders. EPRI Topical Report, NP-5596, Electric Power Research Institute, Palo Alto, CA, January 1988.

[11] Brust FW, Scott P, Rahman S, Ghadiali N, Kilinski T, Francini B, Marschall CW, Miura N, Krishnaswamy P, Wilkowski GM. Assessment of short through-wall circumferential cracks in pipes-experiments and analysis. NUREG/CR-6235, US Nuclear Regulatory Commission, Washington, DC, April 1995.

[12] Firmature R. Elastic-plastic analysis of off-centered cracks in cylindrical structures. A Thesis for Master of Science, Department of Mechanical Engineering, The University of Iowa, Iowa City, IA, July 1998.

[13] Rahman S. A stochastic model for elastic-plastic fracture analysis of circumferential through-wall-cracked pipes subject to bending. Engineering Fracture Mechanics 1995;52(2):265-88.

[14] Hasofer AM, Lind NC. An exact and invariant first-order reliability format. Journal of Engineering Mechanics 1974;100:111-21.

[15] Fiessler B, Neumann HJ, Rackwitz R. Quadratic limit states in structural reliability. Journal of Engineering Mechanics, ASCE 1979;105(EM4):661-76.

[16] Rackwitz R, Fiessler B. Structural reliability under combined random load sequence. Computers and Structures 1978;9:484-94.

[17] Madsen HO, Krenk S, Lind NC. Methods of structural safety. Englewood Cliffs, NJ: Prentice-Hall, 1986.

[18] Hohenbichler M. New light on first- and second-order reliability methods. Structural Safety 1987;4:267-84.

[19] Rubinstein RY. Simulation and the Monte Carlo method. New York: Wiley, 1981.

[20] Harbitz A. An efficient sampling method for probability of failure calculation. Structural Safety 1986;3(1):109-15.

[21] Ibrahim Y, Rahman S. Reliability Analysis of Uncertain Dynamic Systems Using Importance Sampling. In: Proceedings of the Sixth International Conference on Applications of Statistics and Probability in Civil Engineering, Mexico City, Mexico, 1991.

[22] Hohenbichler M. Improvement of second-order reliability estimates by importance sampling. Journal of Engineering Mechanics, ASCE 1988;114(12):2195-9.

[23] Rahman S, Ghadiali N, Paul D, Wilkowski G. Probabilistic pipe fracture evaluations for leak-rate-detection applications. NUREG/ CR-6004, US Nuclear Regulatory Commission, Washington, DC, April 1995.

[24] Ghadiali N, Rahman S, Choi YH, Wilkowski G. Deterministic and probabilistic evaluations for uncertainty in pipe fracture parameters in leak-before-break and in-service flaw evaluations. NUREG/CR-6443, US Nuclear Regulatory Commission, Washington, DC, June 1996. 